\title{
Constructing a cosmological model-independent Hubble diagram of type Ia supernovae with cosmic chronometers
}

\author{
Zhengxiang $\mathrm{Li}^{1 *}$, J. E. Gonzalez ${ }^{2 \dagger}$, Hongwei $\mathrm{Yu}^{3 \ddagger}$, Zong-Hong $\mathrm{Zhu}^{1 \S}$, and J. S. Alcaniz ${ }^{2}$ \\ ${ }^{1}$ Department of Astronomy, Beijing Normal University, Beijing 100875, China \\ ${ }^{2}$ Observatório Nacional, 20921-400, Rio de Janeiro, RJ, Brazil \\ ${ }^{3}$ Center of Nonlinear Science and Department of Physics, \\ Ningbo University, Ningbo, Zhejiang 315211, China
}

\begin{abstract}
We apply two methods, i.e., the Gaussian processes and the non-parametric smoothing procedure, to reconstruct the Hubble parameter $H(z)$ as a function of redshift from 15 measurements of the expansion rate obtained from age estimates of passively evolving galaxies. These reconstructions enable us to derive the luminosity distance to a certain redshift $z$, calibrate the light-curve fitting parameters accounting for the (unknown) intrinsic magnitude of type Ia supernova (SNe Ia) and construct cosmological model-independent Hubble diagrams of SNe Ia. In order to test the compatibility between the reconstructed functions of $H(z)$, we perform a statistical analysis considering the latest SNe Ia sample, the so-called JLA compilation. We find that, for the Gaussian processes, the reconstructed functions of Hubble parameter versus redshift, and thus the following analysis on SNe Ia calibrations and cosmological implications, are sensitive to prior mean functions. However, for the non-parametric smoothing method, the reconstructed functions are not dependent on initial guess models, and consistently require high values of $H_{0}$, which are in excellent agreement with recent measurements of this quantity from Cepheids and other local distance indicators.
\end{abstract}

PACS numbers:

\section{INTRODUCTION}

The inherent relation between the peak luminosity of type Ia supernovae (SNe Ia) and the speed of luminosity evolution after maximum light (known as the Phillips relationship) 1], makes possible to standardize these events as a distance indicator and measure the geometry and dynamics of the universe. As well known, several years after the discovery of the Phillips relationship, observations of some dozens of distant $\mathrm{SNe}$ Ia led to the discovery of the cosmic acceleration [2 4] $]$. In Einstein's general relativity, such behavior implies either the existence of a new field, the so-called dark energy (see Refs. [5, 6] for recent reviews), or that the matter content of the universe is subject to dissipative processes [7 9$]$. The mysterious cause of the current cosmic acceleration can also be attributed to a modification of the standard theory of gravity itself on cosmologically relevant physical scales $[10-12$.

In the past decade, several groups have put a lot of effort into enlarging the sample size of well-measured $\mathrm{SNe}$ Ia events. At the same time, improvements in precision and assessments of systematic errors have also been accomplished. Recently, several SNe Ia samples containing a large number of SNe Ia events with high quality have been released, such as the Sloan Digital Sky Survey-II Supernova Survey (SDSS-II SN Survey) [13, 14], the Union2 and Union2.1 SN Ia released by the Supernova Cosmological Project (SCP) [15, 16], the first three years of Supernova Legacy Survey (SNLS3) 17, 18], and the joint light-curve analysis (JLA) of SDSS-II and SNLS3 [19, 20].

The distance estimation from SNe Ia data is based on the empirical observation that these events form a homogeneous class whose remaining variability is reasonably well captured by two parameters. One of them describes the time stretching of the light-curve $\left(x_{1}\right)$ whereas the other describes the SNe Ia color at maximum brightness $(c)$. In the latest JLA SNe Ia sample [20], which uses the SALT2 model to

\footnotetext{
*zxli918@bnu.edu.cn

† javierernesto@on.br

¥ hwyu@hunnu.edu.cn

$\S$ zhzhu@bnu.edu.cn

ฯ alcaniz@on.br
} 
reconstruct light-curve parameters $\left(x_{1}, c\right.$, and the observed peak magnitude in rest frame $B$ band $\left.m_{\mathrm{B}}^{*}\right)$, the distance estimator assumes that SNe Ia with identical color, shape, and galactic environment have on average the same intrinsic luminosity at all redshifts. This assumption can be quantified by a linear expression, yielding a standardized distance modulus, which relates to the luminosity distance $d_{\mathrm{L}}$ via $\mu=5 \log \left[\frac{d_{\mathrm{L}}}{\mathrm{Mpc}}\right]+25:$

$$
\mu^{\mathrm{SN}}\left(\alpha, \beta, M_{\mathrm{B}}\right)=m_{\mathrm{B}}^{*}-M_{\mathrm{B}}+\alpha \times x_{1}-\beta \times c,
$$

where $\alpha$ and $\beta$ are nuisance parameters which characterize the stretch-luminosity and color-luminosity relationships, reflecting the well-known broader-brighter and bluer-brighter relationships, respectively. The value of $M_{\mathrm{B}}$ is another nuisance parameter which represents the absolute magnitude of a fiducial SNe and was found to depend on the properties of host galaxies, e.g., the host stellar mass $\left(M_{\text {stellar }}\right)$, although the reason is not completely clear [21, 22]. Here, we follow the procedure in Ref [18] to approximately correct for this effect by a simple step function:

$$
M_{\mathrm{B}}= \begin{cases}M_{\mathrm{B}}^{1}, & \text { if } M_{\text {stellar }}<10^{10} M_{\odot} . \\ M_{\mathrm{B}}^{1}+\Delta_{\mathrm{M}}, & \text { otherwise. }\end{cases}
$$

In general, the light-curve fitting parameters, $\alpha$ and $\beta$, are left as free parameters being determined in the global fit to the Hubble diagram. This treatment results in the dependence of distance estimation on the cosmological model used in the analysis. Thus, implications derived from SNe Ia observations with the light-curve fitting parameters determined in the global fit to the Hubble diagram are somewhat cosmological-model-dependent.

Our goal in this paper is to construct a completely cosmological model-independent Hubble diagram of SNe Ia using observational data of the so-called cosmic chronometers [23], where the cosmic expansion rates $H(z)$ are measured from age estimates of red galaxies without any assumption of cosmology.

\section{METHODOLOGY}

The expansion rate, $H=\dot{a} / a$ where $a=1 /(1+z)$, at redshifts $z \neq 0$ can be obtained from the derivative of redshift with respect to cosmic time, i.e., $H(z) \simeq-\frac{1}{1+z} \frac{\Delta z}{\Delta t}$. The difficult task here is to estimate the change in the age of the Universe as a function of redshift $\Delta t$. This can be done by calculating the age difference between two luminous red galaxies at different redshifts, as proposed in Ref. [23]. This method is usually referred to as differential age and the passively evolving galaxies from which $\Delta t$ is estimated are called cosmic chronometers. Currently, 21 measurements of $H(z)$ based on this method (in the redshift range $0.070 \leq z \leq 1.965$ ) have been obtained 24 28]. Although cosmological model-independent, these estimates rely on stellar population synthesis models whose influence on $\Delta t$, according to Ref. [29], becomes important at $z \gtrsim 1.2$. In our analysis, we follow Ref. 30] and consider only $15 H(z)$ measurements up to $z<1.2$ which, in practice, given the redshift distribution of the $H(z)$ data, means $z \leq 1.037$. We also increase slightly $(20 \%)$ the error bar of the highest- $z$ point to account for the uncertainties of the stellar population synthesis models. This ensures that the evolution of the Hubble parameter as a function of redshift reconstructed in this paper is neither dependent on the cosmology nor on the stellar population model.

\section{A. Distances from $\mathbf{H}(\mathrm{z})$ measurements}

Recently, $H(z)$ measurements were used to estimate distances by solving numerically the comoving distance integral for non-uniformly spaced data with a simple trapezoidal rule [31],

$$
d_{c}=c \int_{0}^{z} \frac{d z^{\prime}}{H\left(z^{\prime}\right)} \approx \frac{c}{2} \sum_{i=1}^{N}\left(z_{i+1}-z_{i}\right)\left(\frac{1}{H_{i+1}}+\frac{1}{H_{i}}\right)
$$


The uncertainty associated to the $i^{\text {th }}$ bin is standardly propagated from the errors of $H(z)$ data,

$$
s_{i}=\frac{c}{2}\left(z_{i+1}-z_{i}\right)\left(\frac{\sigma_{H_{i+1}}^{2}}{H_{i+1}^{4}}+\frac{\sigma_{H_{i}}^{2}}{H_{i}^{4}}\right),
$$

and then the error of the integral (3) from $z=0$ to $z=z_{n}$ is $\sigma_{n}^{2}=\sum_{i=1}^{n}=s_{i}^{2}$. Naturally, the precision of this simple trapezoidal rule is sensitive to the uniformity of the spaced data as well as to the number of data points in a certain spaced range. As indicated in Ref. [32], the relative errors of this method decrease remarkably when the number of intervals averagely spaced in $z=0-1$ increases. Here, we use two methods to reconstruct the evolution of the expansion rate with redshift from cosmic chronometer $H(z)$ measurements, namely, Gaussian Processes (GP) and a non-parametric smoothing (NPS). This procedure enable us to achieve model-independent distance estimates by integrating the inverse of the reconstructed function using the approach of Ref. 31] with a very small and uniform step of $\Delta z=z_{i+1}-z_{i}$ (see Sec. 3).

\section{Gaussian processes}

As a powerful non-linear interpolating tool, the Gaussian processes allow us to reconstruct a function from data without assuming a model or parameterization for it. This method has been first pioneered for cosmology [33, 34], and then has been widely used for several purposes, for instance, reconstructions of the equation of state of dark energy [35], cosmography [36, 37], null tests of the concordance model [38, 39], $H_{0}$ from cosmic chronometer data [40], and reconstructions of the distance-duality relation [41].

The reconstruction is based on a mean function with Gaussian error bands, where the function value at $z$ is not independent of the function value at some other point $\tilde{z}$ (especially when $z$ and $\tilde{z}$ are close to each other) and they are related through a covariance function $k(z, \tilde{z})$. This covariance function depends on a set of hyperparameters and there is a wide range of possible candidates for it. As the function of Hubble parameter versus redshift is expected to be infinitely differentiable, we consider the squared exponential covariance function:

$$
k(z, \tilde{z})=\sigma_{f}^{2} \exp \left\{-\frac{(z-\tilde{z})^{2}}{2 l^{2}}\right\},
$$

where the two hyperparameters $\sigma_{f}$ and $l$ are, respectively, related to typical changes in the function value and to the length scale one needs to move in input space to get significant change in the function value. In order to obtain the value of the function, the hyperparameters should be trained by maximizing the marginal likelihood which only depends on the locations of the observations. As the key steps summarized in Ref. [35], another issue in constructing the GP is to decide a prior mean function. In order to achieve an unbiased reconstruction, we should choose a flat prior by taking a constant mean function into consideration. Moreover, it is reasonable and safe to decide a constant function when we do not have any prior information about the reconstructed result. As it was initiated and widely used in the literature, the best choice is a constant mean function because any prior model may introduce significant bias in the results. However, it is also necessary to check the dependence of the reconstruction and following implications on the selected prior mean function. Here, we do it by using different best-fit models, i.e., Einstein-de Sitter (E-D) model, $\Lambda \mathrm{CDM}$, and $\mathrm{wCDM}$ as the prior mean function. Results are summarized in Table【.

In this work, we reconstruct the Hubble parameter as a function of the redshift from $15 H(z)$ measurements of cosmic chronometers by using the GaPP (Gaussian Processes in Python) ${ }^{1}$ [35].

\footnotetext{
${ }^{1}$ http://www.acgc.uct.ac.za/ seikel/GAPP/index.html
} 


\section{Non-parametric smoothing method}

In order to verify the influence of the reconstructing methods on the results, we also use the nonparametric procedure of Ref. 42 45] to reconstruct the $H(z)$ function from cosmic chronometer data. The smoothing function taking into account the data errors is given by

$$
H^{s}(z, \Delta)=H^{g}(z)+N(z) \sum_{i} \frac{\left[H\left(z_{i}\right)-H^{g}\left(z_{i}\right)\right]}{\sigma_{H\left(z_{i}\right)}^{2}} \times \mathcal{K}\left(z, z_{i}\right),
$$

where $H^{s}(z, \Delta), H^{g}\left(z_{i}\right), H\left(z_{i}\right), \sigma_{H}\left(z_{i}\right)$ and $\Delta$ correspond, respectively, to the smoothed data, the initial guess model, the observed data, the error associated with the $H\left(z_{i}\right)$ data and the smoothing scale. The function $N(z)$ is the normalization factor given by:

$$
N(z)^{-1}=\sum_{i} \frac{\mathcal{K}\left(z, z_{i}\right)}{\sigma_{H\left(z_{i}\right)}^{2}}
$$

Given the arbitrariness in the choice of the kernel $\mathcal{K}\left(z, z_{i}\right)$, we tested a Gaussian function and the lognormal kernel of Ref. [42]. We found a small difference between the reconstructed functions from both approaches. In what follows, we will adopt a Gaussian kernel.

We perform a boot-strapping method applying iterative smoothing functions. In the first interaction, we subtract an initial guess model to the data in order to smooth the noise. Then we add back the initial guess model. In the next smoothing, we replace $H^{g}\left(z_{i}\right)$ by the previous smoothed $H^{s}(z)$ and calculate the $\chi^{2}$ value in each iteration. We stop the process when the variation of the $\chi^{2}$ value between two successive steps is $\sim 0.002 \%$ ( $\simeq 1000^{\text {th }}$ iteration $)$.

The reconstruction depends on the value of $\Delta$. For example, for values of $\Delta<0.6$ the reconstructed function has many bumps, for $\Delta$ between [0.6, 0.9] bumps disappear but the reconstruction at high redshift is strongly dependent on the $\Delta$ value whereas for $\Delta>1.0$ the reconstruction in the whole redshift interval considered is weakly dependent on the $\Delta$ value. However, very high values are meaningless because data points at high redshift should not be highly correlated with low redshift data points. In order to select the smoothing scale we minimize over $\Delta$ the cross-validation function, which is defined by:

$$
C V(\Delta)=\frac{1}{n} \sum_{i}\left(H\left(z_{i}\right)-H_{-i}^{s}\left(z_{i} \mid \Delta\right)\right)^{2}
$$

where $H_{-i}^{s}\left(x_{i} \mid \Delta\right)$ denotes the reconstructed Hubble parameter at $z=z_{i}$ without taking into account the ponte $\left(z_{i}, H\left(z_{i}\right)\right)$. For our $15 H(z)$ data discussed in the previous section the $\Delta$ value that asymptotically minimizes Eq. (8) is 1.4 .

As reported in Ref. [42], the initial guess model is not relevant since the reconstruction does not depend on it. Here, we also have tested such dependence by considering different best-fit model, namely, the E-D, $\Lambda \mathrm{CDM}$, and ${ }_{\mathrm{wCDM}}$ as the initial guess one. Results are summarized in Table II An important difference between the analysis reported here and the one performed in Ref. [42] is that in the latter the iteration number of the boot-strapping process $n$ is the free parameter relative to which a $\chi^{2}$ function is minimized to define the $1 \sigma$ region $\left(\chi^{2}=\chi_{m i n}^{2}+1\right)$. In our analysis, in order to calculate the $1 \sigma$ confidence level we extrapolate the technique developed in [46] for linear smoothing and consider the expression:

$$
\sigma_{H^{s}(z)}=\left(\sum_{i} v_{i}^{2} \hat{\sigma}^{2}\right)^{1 / 2}
$$

where $\sigma_{H^{s}(z)}$ is the $1 \sigma$ error of the reconstruction, $v_{i}$ is the smoothing factor $\left(v_{i}=N(z) \mathcal{K}\left(z, z_{i}\right) / \sigma_{H\left(z_{i}\right)}^{2}\right)$ and $\hat{\sigma}^{2}$ is the estimate of the error variance given by

$$
\hat{\sigma}^{2}=\frac{\sum_{i}\left(H\left(z_{i}\right)-H^{s}\left(z_{i}\right)\right)^{2}}{\sum_{j}\left(1-v_{j}\left(z_{j}\right)\right)} .
$$



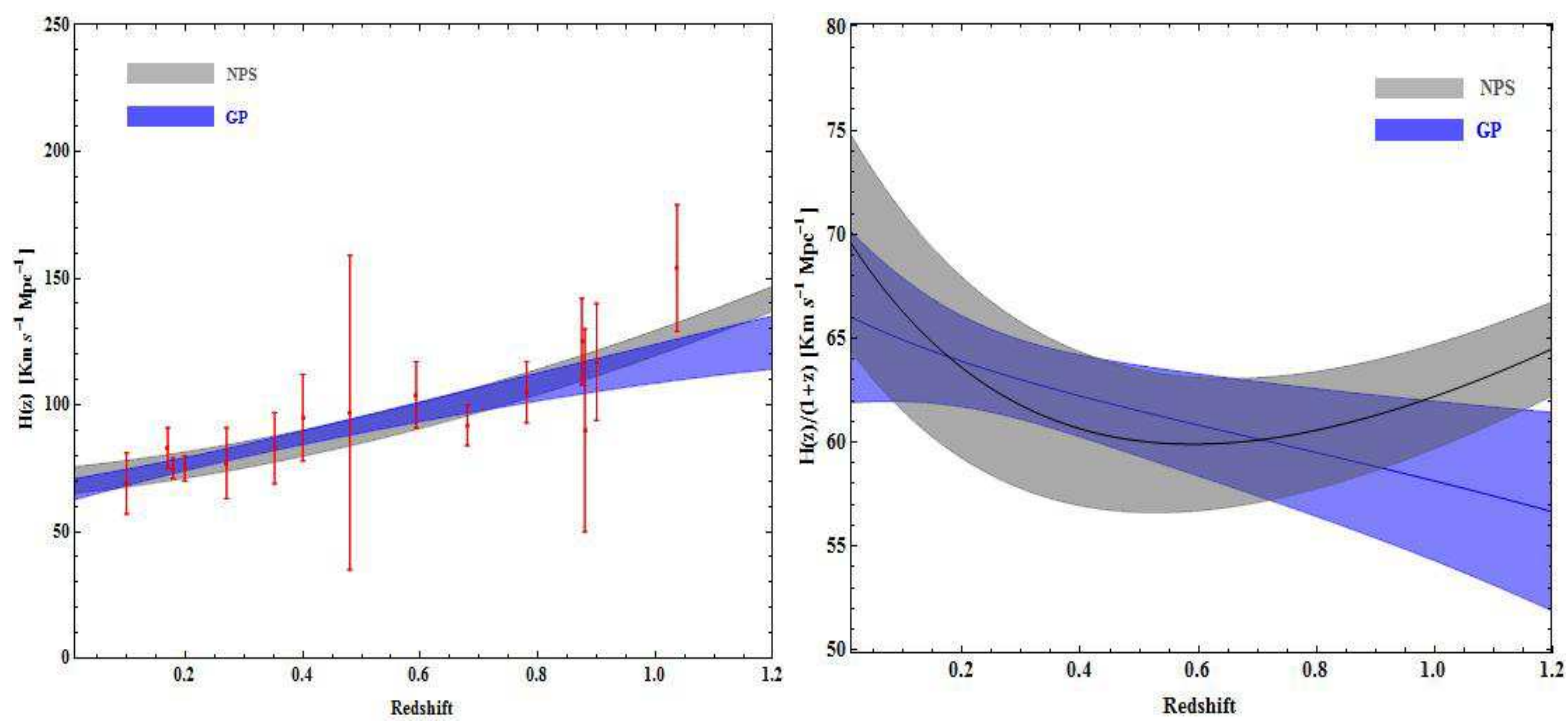

FIG. 1: a) Reconstructions of the Hubble expansion from cosmic chronometer data using Gaussian processes (blue region) with the prior mean function being a constant and non-parametric smoothing method (gray region) with the initial guess model being the best-fit $\Lambda$ CDM. b) The quantity $H(z) /(1+z)$ as a function of $z$ for both reconstructions.

In order to test the validity of this extrapolation, we simulate $H(z)$ data using the $\Lambda$ CDM scenario as fiducial model with different values of $\sigma\left(\sigma_{\text {sim }}\right)$ and compare them with those $\left(\sigma_{\text {rec }}\right)$ calculated from Eq. (9). We find that $\sigma_{r e c}$ is $\simeq 30 \%$ smaller than $\sigma_{r e c}$. We, therefore, add $30 \%$ to the $\sigma_{H^{s}(z)}$ obtained from Eq. (9).

Figure 1a shows both GP and NPS reconstructions of the expansion history from the cosmic chronometer data using the best-fit values for the hyperparameters (GP) and $\Delta$ (NPS). Here, results correspond to reconstruction with the prior mean function being a constant for GP and that with the initial guess model being the best-fit $\Lambda$ CDM for NPS, respectively. For completeness, we also plot $H(z) /(1+z)$ as a function of $z$ in Figure 1b. Clearly, the NPS reconstruction shows a minimum (deceleration/acceleration transition) at $z_{t} \sim 0.6$ whereas the GP function presents no transition in the $z$ interval considered. This could be understood as the latter reconstruction might provide a more reasonable reconstruction and better calibration to the JLA sample if we believe that the transition from deceleration to acceleration has happened in the real case (also in the standard $\Lambda$ CDM model).

\section{B. Calibrations for the light-curve fitting parameters}

In order to calibrate the light-curve fitting parameters and construct cosmological model-independent Hubble diagrams for the JLA SN Ia sample, we first transform the $H(z)$ reconstructions above into distance following the approach proposed in Ref. [31]. Assuming a spatially flat universe, we solve numerically the distance integral and calculate the corresponding uncertainty with a uniform step, $\Delta z=$ $z_{i+1}-z_{i}=0.005$. For completeness, we check the difference between the results from this numerical treatment and the analytical calculation considering a given cosmology. At the redshift range considered, $z<1.2$, and assuming the standard $\Lambda \mathrm{CDM}$ model, the difference in distance modulus is $<0.003 \mathrm{mag}$, which is negligible when compared to the uncertainties of current SNe Ia observations $\left(\simeq 10^{-1} \mathrm{mag}\right)$.

Since the expansion rate measurements obtained from the derivative of redshift with respect to cosmic time, i.e., cosmic chronometer, and the non-parametric methods are cosmology free, distances derived from the reconstructed functions of Hubble parameter with respect to redshift are considered to be the 
true ones, $d_{\mathrm{L}}^{\text {true }}$ or $\mu^{\text {true }}$ (luminosity distance can be obtained from the comoving distance by multiplying $\left.(1+z): d_{L}=(1+z) d_{c}\right)$. And then, $\alpha$ and $\beta$ are fitted using the standard minimum- $\chi^{2}$ route to analytically marginalize the absolute magnitude of a fiducial SNe Ia, $M_{\mathrm{B}}$,

$$
\chi^{2}\left(\alpha, \beta, M_{\mathrm{B}}\right)=A-2 \times M_{\mathrm{B}} \times B+M_{\mathrm{B}}^{2} \times C,
$$

where

$$
\begin{aligned}
& A(\alpha, \beta)=\sum_{i=1}^{740} \frac{\left[\mu^{\mathrm{SN}}\left(z_{i}, \alpha, \beta ; M=0\right)-\mu_{\text {true }}\left(z_{i}\right)\right]^{2}}{\sigma_{\text {tot }, i}^{2}(\alpha, \beta)}, \\
& B(\alpha, \beta)=\sum_{i=1}^{740} \frac{\left[\mu^{\mathrm{SN}}\left(z_{i}, \alpha, \beta ; M=0\right)-\mu_{\text {true }}\left(z_{i}\right)\right]}{\sigma_{\text {tot }, i}^{2}(\alpha, \beta)} \\
& B(\alpha, \beta)=\sum_{i=1}^{740} \frac{1}{\sigma_{\text {tot }, i}^{2}(\alpha, \beta)} .
\end{aligned}
$$

Here $\sigma_{\text {tot }}^{2}$ are propagated from both the statistical uncertainties in SNe Ia and those in the derived $\mu^{\text {true }}$. $\chi^{2}\left(\alpha, \beta, M_{\mathrm{B}}\right)$ in the Eq. (11) has a minimum at $M_{\mathrm{B}}=B / C$ [47], and it is

$$
\widetilde{\chi}^{2}(\alpha, \beta)=A(\alpha, \beta)-\frac{B(\alpha, \beta)^{2}}{C(\alpha, \beta)} .
$$

Therefore, by minimizing $\widetilde{\chi}^{2}(\alpha, \beta)$, we can achieve calibrations for $\alpha$ and $\beta$ with true distances derived from cosmic chronometer observations. On the other hand, we also can benefit an estimation for the nuisance parameter, $M_{\mathrm{B}}$, from this fitting. It should be noted that this merit enable us to break the degeneracy between the Hubble constant $H_{0}$ and the absolute magnitude $M_{\mathrm{B}}$ when we investigate cosmological implications of this Hubble diagram in the following analysis.

\section{RESULTS}

The light-curve fitting parameters calibrated from the reconstructions of $H(z)$ with different prior mean functions for GP and initial guess models for NPS taken into consideration are summarized in Table 【 and Table 【, respectively. As shown in Table I, it is suggested that GP reconstructions and following calibrations and cosmological implications are somewhat sensitive to the assumption of the prior mean function. However, from the Table II] it is found that NPS reconstructions and following results are not dependent on the initial guess model. The difference between the distance modulus $\mu(z)$ derived from the reconstructed $H(z)$ functions with the GP and NPS presented in Figure 1a, and the $\Lambda$ CDM values is shown in Figure 2. In agreement with the results of Figure 1b, the NPS values result in a Hubble diagram more consistent with the $\Lambda \mathrm{CDM}$ prediction than do the GP values. Here, again, it should be noted that, rather than fixing $H_{0}=70 \mathrm{~km} \mathrm{~s}^{-1} \mathrm{Mpc}^{-1}$ to obtain the values of $M_{\mathrm{B}}^{1}$ and $\Delta_{M}$ in the global fit for the $\Lambda \mathrm{CDM}$ model [20], these values, in our analysis, can be estimated from the cosmologicalmodel-independent calibration by confronting light-curve fitting parameters-dependent distances of $\mathrm{SNe}$ Ia (Eq. 11) with the ones derived from the reconstructed function of $H(z)$.

We also investigate some cosmological implications of these model-independent Hubble diagrams for the JLA sample. Assuming a spatially flat $\Lambda \mathrm{CDM}$ scenario, whose expansion rate is given by $H(z)=$ $H_{0}\left[\Omega_{m}(1+z)^{3}+\left(1-\Omega_{m}\right)\right]^{1 / 2}$, where $H_{0}$ and $\Omega_{m}$ are the present value of the Hubble parameter and matter density parameter, respectively, we derive the constraints on the $\Omega_{m}-H_{0}$ plane and the results are also summarized in Table \and Table II. Once again results based on GP are slightly dependent on the prior mean function. In contrast, results based on NPS are hardly sensitive the initial guess model. Moreover, we also present constrained contours on the $\Omega_{m}-H_{0}$ plane with reconstructed functions in the Figure 1a taken into account. For the Gaussian processes, we obtain that the value of the Hubble constant is fairly compatible with the constraint from the latest Planck+WMAP9+BICEP2 CMB measurements reported in Ref. [48]. For the smoothing method, we found that the constraint on the Hubble constant 


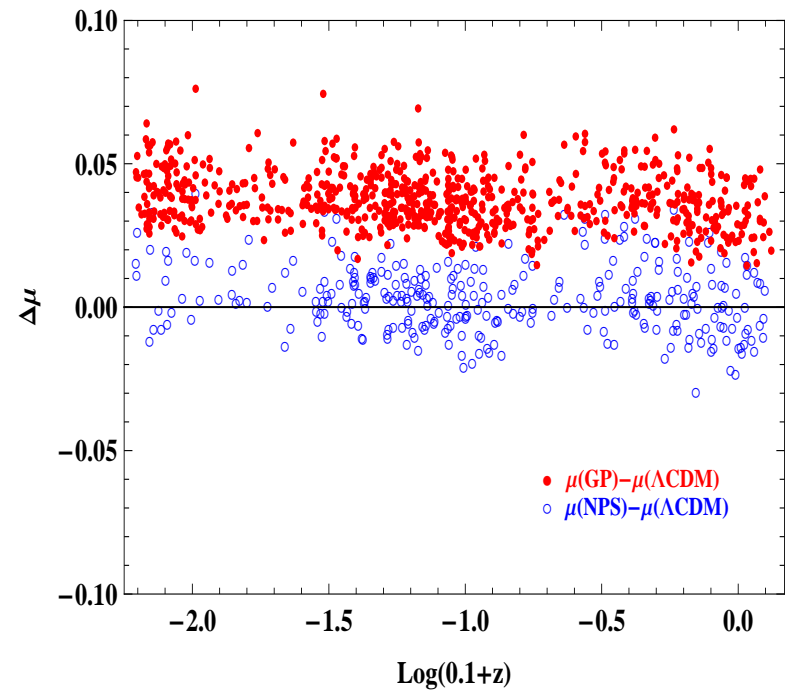

FIG. 2: The estimated distance modulus difference calculated with the GP, NPS and the $\Lambda$ CDM values obtained from the light-curve fitting parameters.

is excellently consistent with the recent measurement of the local Hubble parameter obtained from the recession velocity of objects around us $\left(H_{0}=73.80 \pm 2.40\right.$ at $68.3 \%$ confidence level) [49] (see Figure $\left.3 \mathrm{~b}\right)$.

\begin{tabular}{|c|c|c|}
\hline Prior mean functions & Calibrations on $(\alpha, \beta, M, \Delta M)$ & Constraints on $\Omega_{m}$ and $H_{0}$ \\
\hline Constant & $0.137,3.036,-19.081,-0.056$ & $0.291_{-0.027}^{+0.029}, 68.924_{-0.477}^{+0.476}$ \\
\hline E-D & $0.149,3.157,-19.042,-0.029$ & $0.270_{-0.027}^{+0.028}, 70.838_{-0.494}^{+0.493}$ \\
\hline$\Lambda$ CDM & $0.158,3.439,-18.983,-0.031$ & $0.255_{-0.028}^{+0.029}, 72.795_{-0.522}^{+0.521}$ \\
\hline wCDM & $0.152,3.307,-19.053,-0.042$ & $0.267_{-0.028}^{+0.029}, 70.216_{-0.498}^{+0.497}$ \\
\hline
\end{tabular}

TABLE I: Summary of the results with different prior mean functions for the GP.

\begin{tabular}{|c|c|c|}
\hline Initial guess models & Calibrations on $(\alpha, \beta, M, \Delta M)$ & Constraints on $\Omega_{m}$ and $H_{0}$ \\
\hline $\mathrm{E}-\mathrm{D}$ & $0.154,3.190,-19.022,-0.019$ & $0.264_{-0.027}^{+0.028}, 71.738_{-0.501}^{+0.500}$ \\
\hline$\Lambda \mathrm{CDM}$ & $0.155,3.221,-19.014,-0.017$ & $0.261_{-0.027}^{+0.028}, 72.070_{-0.505}^{+0.504}$ \\
\hline $\mathrm{wCDM}$ & $0.153,3.172,-19.028,-0.021$ & $0.265_{-0.027}^{+0.028}, 71.525_{-0.499}^{+0.498}$ \\
\hline
\end{tabular}

TABLE II: Summary of the results with different initial guess models for the NPS.

\section{CONCLUSIONS}

As is well known, implications derived from current SNe Ia analyses, where the light-curve fitting parameters are usually determined to the global fit in the frame of the standard dark energy model, are cosmological-model-dependent. In this paper, we have applied two non-parametric methods to reconstruct the Hubble expansion using $15 H(z)$ measurements $(z \leq 1.2)$ from cosmic chronometers and transformed 

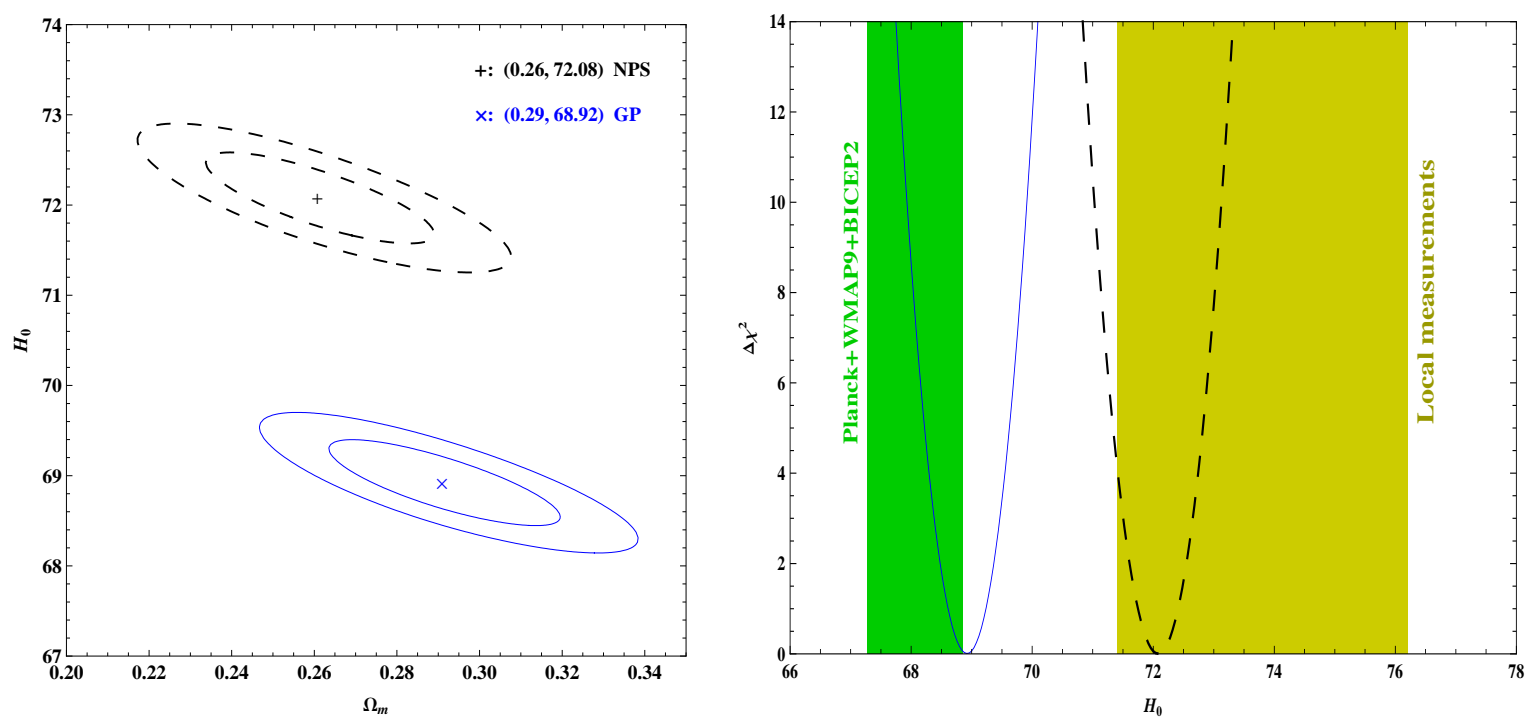

FIG. 3: Left: Constraints on the spatially flat $\Lambda$ CDM scenario using the model-independent Hubble diagrams of the JLA compilation. Right: Marginalized distribution of the local Hubble parameter $H_{0}$ from the $H(z)$-calibrated Hubble diagrams of JLA sample using Gaussian processes (blue solid) and the non-parametric smoothing method (black dashed). The dark green and dark yellow rectangles correspond to the $68.3 \%$ interval of $H_{0}$ obtained from the latest Planck+WMAP9+BICEP2 CMB [48] and Cepheids observations [49], respectively.

the reconstructed functions into distances by carrying out a numerical integration. The choice of this reduced $H(z)$ sample is based on the arguments of Refs. [29, 30], which ensures that the evolution of the Hubble parameter reconstructed in our analysis is neither dependent on the cosmology nor on the stellar population model.

By using the derived model-independent distances we have calibrated the light-curve fitting parameters and constructed a completely cosmological model-independent Hubble diagram for the JLA sample. The results suggest that the uncertainties on the light-curve fitting parameters obtained from the $H(z)$ reconstructions are almost of the same order of magnitude as the ones determined in the global fit for the $\Lambda$ CDM model. Therefore, we expect the constraining power of any analysis derived from these $H(z)-$ calibrated Hubble diagrams to be nearly identical to the one obtained when the global fit to a given model is performed. It should be emphasized, however, that cosmological implications of the Hubble diagrams constructed from $H(z)$ data do not suffer with cosmological model-dependence.

However, we have shown that these diagrams and their implications depend reasonably on the method used to reconstruct the Hubble evolution. Furthermore, for Gaussian processes, reconstruction and following analysis are obviously sensitive to the prior mean function. Assuming the spatially flat $\Lambda$ CDM model, we have derived constraints on the matter density parameter $\Omega_{m}$ and Hubble constant $H_{0}$ from the JLA sample. For the analysis that uses Gaussian processes, it is shown that constraints on model parameters, $\Omega_{m}$ and $H_{0}$, vary obviously when different prior mean functions are considered. In the reasonable and safe case with the prior mean function being constant, it is shown that the constraint on $H_{0}$ is quite compatible with that derived from the latest Planck+WMAP9+BICEP2 CMB observations at $68.3 \%$ confidence level. When the non-parametric smoothing procedure is applied, results are hardly dependent on the initial guess model and consistently favor a higher value of $H_{0}$, which are in excellent agreement with the recent local measurement of the expansion rate from Cepheids observations.

A final aspect worth emphasizing is that, differently from the GP reconstruction, the expansion rate $H(z)$ obtained from the smoothing method behaves similarly to the one predicted by the $\Lambda$ CDM model, with the deceleration/acceleration transition happening around $z \simeq 0.6$ (Figure 1b). Therefore, taking the standard evolution as a good description for the late-time evolution of the Universe, the NPS results 
obtained in this analysis seem to be more consistent than those derived from the GP method.

\section{Acknowledgments}

Zhengxiang Li, Hongwei Yu, and Zong-Hong Zhu are supported by the Ministry of Science and Technology National Basic Science Program (Project 973) under Grants Nos. 2012CB821804 and 2014CB845806, the National Natural Science Foundation of China under Grants Nos. 11505008, 11373014, 11073005, 11375092, and 11435006, the China Postdoc Grant No. 2014T70043, and the Youth Scholars Program of Beijing Normal University. J. E. Gonzalez and J. S. Alcaniz are supported by CAPES, CNPq and FAPERJ (Brazilian Agencies).

[1] M. M. Phillips, ApJ, 413, L105 (1993).

[2] A. G. Riess, et al., AJ, 116, 1009 (1998).

[3] B. P. Schmidt, N. B. Suntzeff, M. M. Phillips, et al., ApJ, 507, 46 (1998).

[4] S. Perlmutter, et al., ApJ, 517, 565 (1999).

[5] R. R. Caldwell, \& M. Kamionkowski, Ann. Rev. Nucl. Part. Sci., 59, 397 (2009).

[6] M. Li, X.-D. Li, S. Wang, \& Y. Wang, Commun. Theor. Phys., 56, 525 (2011).

[7] J. A. S. Lima and J. S. Alcaniz, Astron. Astrophys. 348, 1 (1999)

[8] J. S. Alcaniz and J. A. S. Lima, Astron. Astrophys. 349, 729 (1999)

[9] L. P. Chimento, A. S. Jakubi, D. Pavón \& W. Zimdahl, PRD 67, 083513 (2003).

[10] M. Ishak, A. Upadhye, \& D.N. Spergel, Phys. Rev. D 74, 043513 (2006).

[11] M. Kunz, \& D. Sapone, Phys. Rev. Lett. 98, 121301 (2007).

[12] E. Bertschinger, \& P. Zukin, Phys. Rev. D 78, 024015 (2008).

[13] J. A. Frieman, et al., AJ, 135, 338 (2008).

[14] R. Kessler, et al., ApJS, 185, 32 (2009).

[15] R. Amanullah, C. Lidman, D. Rubin, et al., ApJ, 716, 712 (2010).

[16] N. Suzuki, D. Rubin, C. Lidman, et al., ApJ, 746, 85 (2012).

[17] J. Guy, M. Sullivan, A. Conley, et al., A\&A, 523, A7 (2010).

[18] A. Conley, J. Guy, M. Sullivan, et al., ApJS, 192, 1 (2011).

[19] M. Betoule, J. Marriner, N. Regnault, et al., A\&A, 552, A124 (2013).

[20] M. Betoule, R. Kessler, J. Guy, et al., A\&A, 568, A22 (2014).

[21] M. Sullivan, J. Guy, A. Conley, et al., ApJ, 737, 102 (2011).

[22] J. Johansson, D. Thomas, J. Pforr, et al., MNRAS, 435, 1680 (2013).

[23] R. Jimenez, \& A. Loeb, ApJ, 573, 37 (2002).

[24] R. Jimenez, L. Verde, T. Treu, \& D. Stern, ApJ, 593, 622 (2003).

[25] J. Simon, L. Verde, \& R. Jimenez, PRD, 71, 123001 (2005).

[26] D. Stern, R. Jimenez, L. Verde, M. Kamionkowski, \& S. A. Stanford, JCAP, 2, 8 (2010).

[27] M. Moresco, et al., JCAP, 8, 6 (2012).

[28] M. Moresco, arXiv:1503.01116 [astro-ph.CO]. 
[29] M. Moresco, A. Cimatti, R. Jimenez, L. Pozzetti, G. Zamorani, M. Bolzonella, J.Dunlop, F. Lamareille, et al., JCAP 1208006 (2012).

[30] L. Verde, P. Protopapas, \& R. Jimenez, Phys. Dark Univ. 5-6, 307 (2014).

[31] R. F. L. Holanda, J. C. Carvalho, \& J. S. Alcaniz, JCAP, 04, 027 (2013).

[32] K. Liao, Z. Li, J. Ming, \& Z.-H. Zhu, PLB, 718, 1166 (2013).

[33] T. Holsclaw, et al., PRD 82, 103502 (2010).

[34] T. Holsclaw, et al., PRL, 105, 241302 (2010).

[35] M. Seikel, C. Clarkson, \& M. Smith, JCAP, 6, 36 (2012).

[36] A. Shafieloo, A. Kim, \& E. Linder, PRD 85, 123530 (2012).

[37] A. Shafieloo, A. Kim, \& E. Linder, PRD 87, 023520 (2013).

[38] M. Seikel, S. Yahya, R. Maartens, \& C. Clarkson, PRD, 86, 083001 (2012).

[39] S. Yahya, M. Seikel, C. Clarkson, R. Maartens, \& M. Smith, PRD, 89, 023503 (2014).

[40] V. C. Busti, C. Clarkson, \& M. Seikel, MNRAS, 441, L11 (2014).

[41] Y. Zhang, arXiv: 1408.3897.

[42] A. Shafieloo, U. Alam, V. Sahni, \& A. Starobinsky, MNRAS, 366, 1081 (2006).

[43] A. Shafieloo, MNRAS, 380, 1573 (2007).

[44] A. Shafieloo \& C. Clarkson PRD, 81, 083537 (2010).

[45] A. Shafieloo, JCAP, 1208, 002 (2012).

[46] A. Bowman \& A. Azzalini, Applied Smoothing Techniques for Data Analysis, Oxford University Press Inc., New York, 1997.

[47] M. Goliath, R. Amanullah, P. Astier, A. Goobar, \& R. Pain, A\&A, 380, 6 (2001).

[48] B. Hu, J.-W. Hu, Z.-K. Guo, \& R.-G. Cai, PRD, 90, 023544 (2014).

[49] A. G. Riess, L. Macri, S. Casertano, et al., ApJ, 730, 119 (2011).

[50] B. Santos, J. C. Carvalho, \& J. S. Alcaniz, Astropart. Phys. 35, 17 (2011). 